\title{
Quality of Service Provision and Capacity Expansion through Extended-DSA for $5 \mathrm{G}$
}

\author{
Uwe Herzog $^{1}$, Andreas Georgakopoulos ${ }^{2}$, Ioannis-Prodromos Belikaidis ${ }^{2}$, Michael Fitch ${ }^{3}$, Keith Briggs ${ }^{3}$, Salvador \\ Diaz $^{4}$, Óscar Carrasco ${ }^{4}$, Klaus Moessner ${ }^{5}$, Benoit Miscopein ${ }^{6}$, Shahid Mumtaz \\ ${ }^{1}$ EURESCOM, Heidelberg, Germany, herzog@eurescom.eu \\ ${ }^{2}$ WINGS ICT Solutions, Athens, Greece, \{andgeorg, iobelika $\}$ wings-ict-solutions.eu \\ ${ }^{3}$ BT, Ipswich, UK, \{michael.fitch, keith.briggs $\} @$ bt.com \\ ${ }^{4}$ Sistelbanda SA, Valencia, Spain, \{ sdiaz, ocarrasco $\} @$ sistelbanda.es \\ ${ }^{5}$ University of Surrey, Guildford, UK, k.moessner@surrey.ac.uk \\ ${ }^{6}$ CEA-Leti Minatec, Grenoble, France, benoit.miscopein@cea.fr \\ ${ }^{7}$ Instituto de Telecomunicações, Aveiro, Portugal, smumtaz@av.it.pt
}

\begin{abstract}
G systems are expected to advance on a number of aspects compared to current systems, e.g. providing a $\mathbf{1 0 0 0}$ times higher capacity, a much lower latency and improved quality of user experience. This paper presents the vision and approach followed by the $\mathbf{H 2 0 2 0}$ project SPEED-5G. The approach is based on densification of small cells, exploitation of Multi-RAT, development of new resource management techniques and a more efficient use of spectrum. A novel 5G system architecture is proposed based on the Network Slicing paradigm, which enables a highly flexible, scalable and backwards compatible architecture. A core aspect is the definition of a new MAC layer that facilitates Multi-RAT access and allows prioritising and allocating traffic across heterogeneous access technologies.
\end{abstract}

Keywords - Small cells, Multi-RAT, Network Architecture, Network Slicing, SDN, NFV, eDSA, MAC.

\section{INTRODUCTION}

Requirements for $5 \mathrm{G}$ systems refer to a 1000 times higher capacity [1], 1 ms maximal latency, seamless connectivity across different access technologies and the highest possible Quality of Experience (QoE) for users. To meet those targets, significant operational and infrastructure investments will be required. Unfortunately revenues are not growing at the same rate as costs, as average revenue per user (ARPU) is expected to remain at best constant in mature markets. Moreover the current lack of dynamic control across wireless network resources is leading to unbalanced spectrum loads and a perceived capacity bottleneck. Tackling inefficiencies is therefore one key aspect of the SPEED-5G approach. A major challenge for future networks is to map various types of traffic and Quality of Service (QoS) requirements across the most appropriate radio technologies and spectrum bands, taking into consideration a larger variety of licensing schemes compared to current deployments.

The above mentioned issues are being addressed by the EU-funded research project SPEED-5G [2]. This paper presents the approach and main results achieved so far. SPEED-5G's main objective is to achieve a significantly better exploitation of heterogeneous wireless technologies, providing higher capacity together with the ultra-densification of cellular technology, and effectively supporting the new 5G QoE requirements. The activities focus along three main dimensions, as shown in Fig. 1:
i. ultra-densification through small cells,
ii. additional spectrum,
iii. exploitation of resources across technologies.

This three dimensional model in the rest of the paper is referred to as extended-Dynamic Spectrum Allocation (eDSA). eDSA implies that several spectrum bands, cells and technologies are jointly managed in order to offer improved QoE and a tremendous capacity increase in a cost-efficient manner.

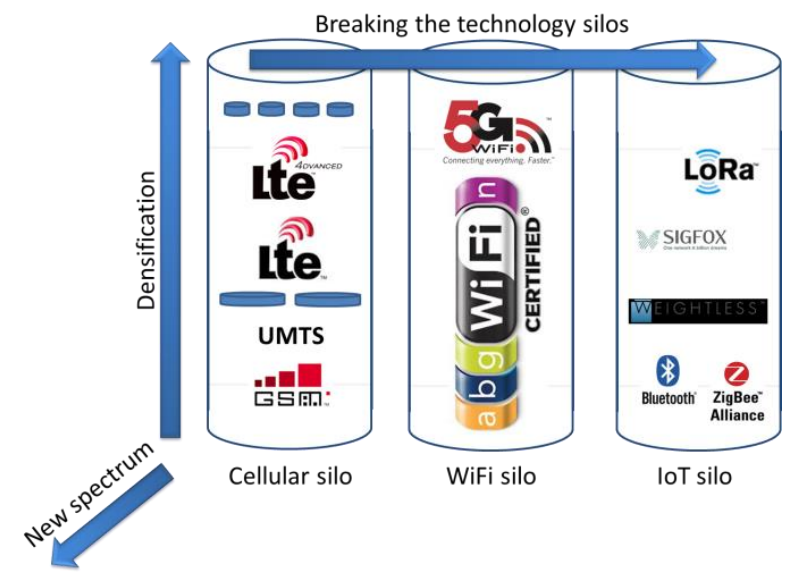

Fig. 1. SPEED-5G degrees of freedom for an optimized management of resources

As stated above, SPEED-5G defines optimised resource management across heterogeneous technologies. As heterogeneity of technologies and "quality" of spectrum (licensed, lightly-licensed or unlicensed) will impact the QoE delivery, SPEED-5G will also analyse the impact of spectrum allocation (in heterogeneous systems) and interference 
limitations (in ultra-dense system) on QoE. The project considers video applications, which represents $70 \%$ of mobile traffic, as a benchmark. On the other hand, Internet of Things (IoT) traffic, which implies a larger number of mostly low bandwidth connections, will be used as another benchmark for testing the multi-RAT techniques proposed by SPEED-5G.

The rest of the paper is organised as follows: Section 2 defines four key use cases for $5 \mathrm{G}$ systems and derives requirements based on the chosen Key Performance Indicators (KPIs). Section 3 presents a first analysis of the $5 \mathrm{G}$ value chain as impacted by the SPEED-5G innovation. Section 4 details the proposed novel 5G system architecture, and uses an instantiation of a particular use case to show how the architecture maps into practical systems. Section 5 presents first results on the Radio Resource Manager, which' task is to select the most appropriate radio access technology. In section 6 a new MAC layer is defined which facilitates MULTI-RAT access - a core element of the SPEED-5G approach. Finally, Section 7 draws the conclusions and hints at the next steps of the project.

\section{SPEED-5G USE CASES AND KPIS}

The main objective of SPEED-5G is to develop novel solutions so as to address 5G technology trends and requirements [3]. Specifically, the main scenarios investigated in SPEED-5G refer to indoor and indoor/ outdoor scenarios (around buildings) where capacity demands are the highest. In these scenarios, eDSA can be a very effective approach exploiting co-operation across technologies and bands, resulting in significant performance improvements. The project uses as reference a revised subset of the use cases proposed by the NGMN association [4] and the METIS project [5].

Fig. 2 depicts the four selected use cases which cover the main 3GPP/NGMN services such as: Massive IoT communications, evolved Mobile Broadband (eMBB), UltraReliable Communications (URC) and High-speed mobility. These use cases cover most of the SPEED-5G concepts for urban communications, IoT, mission critical and vehicular services and as a result are representative for validating RRM/MAC solutions and eDSA techniques that SPEED-5G proposes.

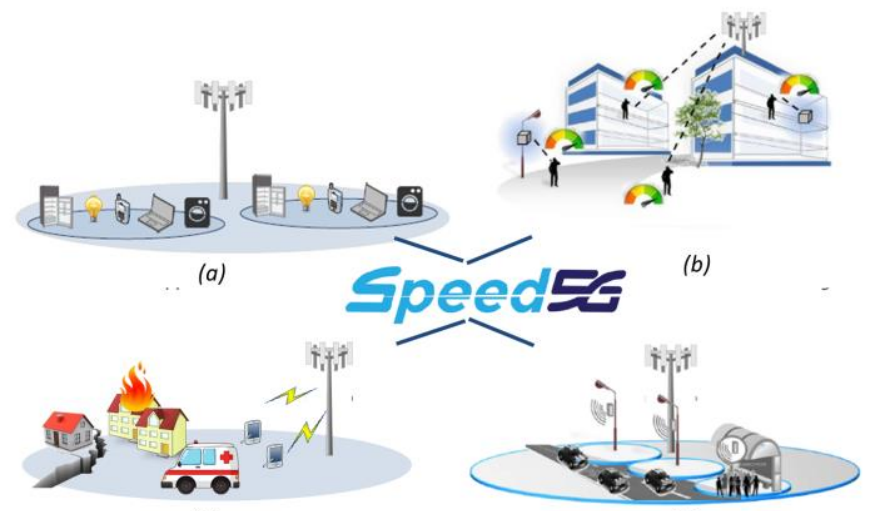

(c)

(d)

Fig. 2. SPEED-5G selected use cases

\section{A. Massive IoT communications}

Compared to the previous network generations, the IoT paradigm will have a major role in $5 \mathrm{G}$ networks. As pointed out by numerous white papers, the seamless integration of IoT in the $5 \mathrm{G}$ network architecture design constitutes one of the main goals of 5G. Furthermore, novel medium access strategies have to be considered in order to be able to deal with the different levels of spectrum licensing (licensed/unlicensed/lightly-licensed bands) and also with the typical fragmented spectrum at low frequencies below $6 \mathrm{GHz}$. Supporting grant-free transmissions can drastically simplify the signalling processing overhead, reducing the power consumption as well.

In this use case, devices can be located in either indoor or outdoor locations, connected to small cells and/or to macro cells, depending on the availability. Small cells can be connected to the network of the operator either using a broadband wired link or a radio link that implements a backhaul connection to the evolved-NodeB (eNB), carrying both data and control data.

\section{B. evolved Mobile Broadband (eMBB)}

This use case refers to a mixture of domestic, enterprise and public access outdoor and indoor environments located in a densely populated urban area. Typical examples can be a block of apartments, a square with shops or a recreation park. In this use case, it is considered that a massive deployment of small cells can provide a uniform broadband experience to users, satisfying their demands for high data rates and very low latency for the smooth operation of applications such as high resolution multimedia streaming, gaming, video calling, and high-bandwidth cloud services. Besides the quality guarantees, connectivity must be ensured for pedestrian users who move from indoor to outdoor environments and vice versa. Finally, an additional challenge refers to the efficient usage of heterogeneous backhaul technologies, ranging from $\mathrm{xDSL}$ and fibre to millimetre-wave connections, used to transport data towards the cells.

To satisfy the $5 \mathrm{G}$ requirements that characterize this use case, novel, efficient MAC and RRM mechanisms are required for an improved usage of the available spectrum (licensed, lightly licensed and unlicensed), and for supporting multiple radio access technologies (RAT). At the same time, this extreme resource reuse may create a rather high level of interference among terminals and between terminals and Base Stations (BS), thus requiring a flexible and enhanced level of coordination to improve the overall network performance.

\section{Ultra-Reliable Communications}

Ultra-Reliable Communication (URC) methods are intended to enable high degrees of network availability, with guarantees on performance metrics such as latency. One of the main objectives of SPEED-5G is to provide scalable solutions for networks supporting services that will meet the extreme $5 \mathrm{G}$ requirements on availability and reliability. 
The reliability and latency of traditional communication systems were designed with the human user in mind. However, future wireless systems will have to support new applications based on machine-to-machine and IoT communications with stringent real-time constraints in order to enable new functionalities e.g. for traffic safety, traffic efficiency or mission-critical control for industrial and military applications. These new applications will require much stricter guarantees in reliability and lower latency levels than the ones provided by traditional wireless systems. A typical example of such application is the monitoring of electricity grids, where shut-down in the case of malfunctions may be required in the time-scale of a few milliseconds.

\section{High-speed mobility}

High-speed mobility is a challenging use case for $5 \mathrm{G}$. SPEED-5G does not aim to serve high-speed trains, but rather focuses on high-speed mobility cars, e.g. when driving on highways. The GSM Association (GSMA) in a recent article [6] claims that "Automated and connected driving will be a pillar of Europe's industrial renaissance".

In this context, SPEED-5G is interested in providing performance evaluation of such use case in order to highlight the benefits of the proposed new solutions, e.g. a novel radio resource management scheme, in high-mobility environments where broadband communications are targeted.

\section{E. KPIs \& Requirements}

TABLE I. provides an overall summary of the main KPIs and requirements that show to be consistent with NGMN's [4] target values for similar scenarios. These KPIs are in line with some of specifications introduced by other organizations (e.g. ITU), for example in latency requirements of certain use cases, but may have different predictions or visions for other KPIs (i.e. connection density).

Also by taking into account that the current commercial deployed systems have usually throughput below 100Mbps, latency of some tens of milliseconds and are unable to efficiently provide stable and continuous network connection in environments characterized with high speed mobility (e.g. up to $500 \mathrm{~km} / \mathrm{h}$ ), we understand the need for more testing and research which must be done in order to converge and achieve the targeted requirements in real environments. As a result, some of the requirements may have been already met under some use cases, but some others need more thorough research (e.g., data rate, support of ultra-dense connection densities, ultra-high mobility).

TABLE I. KPIS AND REQUIREMENTS OF SPEED-5G USE CASES

\begin{tabular}{|c|c|c|c|c|}
\hline \multirow[b]{2}{*}{ KPI } & \multicolumn{4}{|c|}{ Requirements } \\
\hline & Massive IoT communications & $\begin{array}{l}\text { evolved Mobile } \\
\text { Broadband }\end{array}$ & Ultra-reliable communications & High-Speed mobility \\
\hline $\begin{array}{c}\text { User Experienced } \\
\text { Data Rate } \\
\end{array}$ & From tens to hundreds of Kbps & $\begin{array}{l}\text { DL: } 300 \mathrm{Mbps} \\
\text { UL: } 50 \mathrm{Mbps}\end{array}$ & $\begin{array}{l}\text { DL: several kbps upwards } \\
\text { UL: several kbps upwards }\end{array}$ & $\begin{array}{l}\text { DL: 50Mbps } \\
\text { UL: } 25 \mathrm{Mbps}\end{array}$ \\
\hline E2E Latency & Order of seconds or more & $10 \mathrm{~ms}$ & $1 \mathrm{~ms}$ upwards & $10 \mathrm{~ms}$ \\
\hline Mobility & On demand & $\begin{array}{c}\text { On demand, } 0-100 \\
\mathrm{~km} / \mathrm{h}\end{array}$ & Mostly static & $\begin{array}{l}\text { On demand (up to } \\
500 \mathrm{~km} / \text { ) }\end{array}$ \\
\hline $\begin{array}{c}\text { Connection } \\
\text { Density }\end{array}$ & Up to 200,000 devices $/ \mathrm{km}^{2}$ & $200-2500 / \mathrm{km}^{2}$ & Mostly low, but variable & $\sim 2000 / \mathrm{km}^{2}$ \\
\hline Traffic Density & Not critical & $\begin{array}{l}\text { DL: } 750 \mathrm{Gbps} / \mathrm{km}^{2} \\
\text { UL: } 125 \mathrm{Gbps} / \mathrm{km}^{2}\end{array}$ & Highly variable & $\begin{array}{l}\text { DL: } 100 \mathrm{Gbps} / \mathrm{km}^{2} \\
\text { UL: } 50 \mathrm{Gbps} / \mathrm{km}^{2}\end{array}$ \\
\hline
\end{tabular}

\section{I. SPEED-5G VALUE CHAIN ANALYSIS}

SPEED-5G aims at breaking up the conventional wireless technologies framework by proposing a novel three dimensional eDSA model. This model is being investigated under indoor and indoor/outdoor scenarios where capacity demands are at the highest, but also where the eDSA is most effective at exploiting co-operation across technologies and bands. The analysis of the eDSA model under different scenarios is valuable for key stakeholders to decide not only their positions in the telecommunication market, but also business aspects arising from SPEED-5G key notions. These stakeholders are: operators, vendors, end users, services provider and regulators.

Operators: Operators will use the results of SPEED-5G to strengthen their positions in European collaborative research in telecommunication by acquiring knowledge in the important area of capacity increase by using small cells in 5G networks. For example, the 'home-hub' series of domestic small cells will be developed to have LTE added, but the roadmap beyond this depends on the success of flexible layer 1 and 2 as results from SPEED-5G. Moreover, operators will also use the results of SPEED-5G to move more vigorously and confidently into adjacent markets, such as IoT, both in building and for longer range. Finally, SPEED-5G outcome shall also identify possible gaps that need further research and this awareness will be used to steer future research directions of the telecom operators.

Vendors: Vendors will play a very important role as one of the key stakeholders for SPEED-5G. Vendors will be encouraged to make new devices which will be compatible to SPEED-5G technologies. For example, these new devices will have 
multiple MAC interfaces (i.e. FBMC, OFDMA etc.) to download gigabytes of data faster than with currently available devices. New SPEED-5G devices will also lead to significantly enhancements in augmented reality, industry automation, and wearable devices.

End-users: End users will become the true beneficiaries of SPEED-5G and will experience a completely distinctive user experience in term of "Mobile Internet" over Speed-5G Heterogeneous Networks (HetNets)

Service providers: Service providers can utilize new features and services that SPEED-5G can provide, and deliver amazing infotainment services.

Regulators: There is growing interest in Dynamic Spectrum Access (DSA) from regulatory agencies (e.g. FCC, Ofcom) and a variety of industry standardisation forums including IEEE SCC41, 802.11y, and 802.22. The SPEED-5G approach enables real-time optimization of spectral resources and radically changes spectrum management that depends on preplanned frequency assignments. SPEED-5G targets bands below $5 \mathrm{GHz}$ which is also the main target of many regulators before next WRC in 2018. The regulatory stipulations for $5 \mathrm{G}$ technologies must be taken care of by the regulatory bodies. Although 5G technologies are in developing and evolving process it ought to be regularized for the betterment of deployment. According to [7][8], the following aspects should be addressed in $5 \mathrm{G}$ regulation:

i. Location Accuracy $\rightarrow$ more advanced accurate location finder in $5 \mathrm{G}$.

ii. Lawful Intercept $\rightarrow$ the technical challenge is to develop a $5 \mathrm{G}$ architecture that enables communications not necessarily passing over centralized network elements, whilst ensuring compliance with lawful intercept regulations.

iii. Tower sharing $\rightarrow$ the $5 \mathrm{G}$ architecture should support solutions to minimize the number of antennas in shared multi-RAT environments.

iv. Flexible Spectrum Use $\rightarrow$ spectrum licenses should be flexible enough to allow operators to meet the rollout demand while being capable of using $5 \mathrm{G}$ spectrum for backhaul when and where appropriate.

v. Accessibility $\rightarrow$ Broadband wireless services are becoming part and parcel of our daily life, so $5 \mathrm{G}$ services must have easy access to physically challenged people.

\section{SPEED-5G ARCHITECTURE}

Although HetNet deployment has been extensively investigated in the last 3GPP releases and network densification is seen as a pillar of the future $5 \mathrm{G}$ systems, small cells are not natively supported in current LTE-based approaches.

The SPEED-5G project proposes a novel architecture focus on small cells and its particular characteristics and challenges. The challenges are mainly related to the expected high number of small cell being SON, the efficient spectrum usage and its management key features. These functionalities need to be addressed by design.

In order to satisfy the capacity requirements of future wireless services and maximize the macro cell offloading, solutions able to provide more efficient spectrum usage are required. On the one hand, it is necessary to take full advantage of available spectrum by dynamically access, licensed, licensed shared, and license-exempt bands. On the other hand, enhanced interference coordination mechanisms have to be designed to enable fair coexistence on frequency resources and maintaining high QoE. To achieve these goals and reduce the complexity of network planning and deployment phases, the envisioned $5 \mathrm{G}$ architecture should allow for easy and autonomous management, configuration, and optimization of small cells. Finally, a lean and scalable design characterized by limited interfaces is required to easily manage a high number of small cells, avoid signalling congestion, and improve the system efficiency.

As previously expressed, SPEED-5G supports eDSA which aims at managing several RATs and offering an efficient means of offloading part of traffic of the considered $5 \mathrm{G}$ use-cases, considering various shared license regimes.

The SPEED-5G proposed architecture (Fig. 3) follows the most recent software trends, where traditional networks are replaced by logical networks deployed over slices [9].

\section{SPEED-5G Edge Virtualized Architecture}

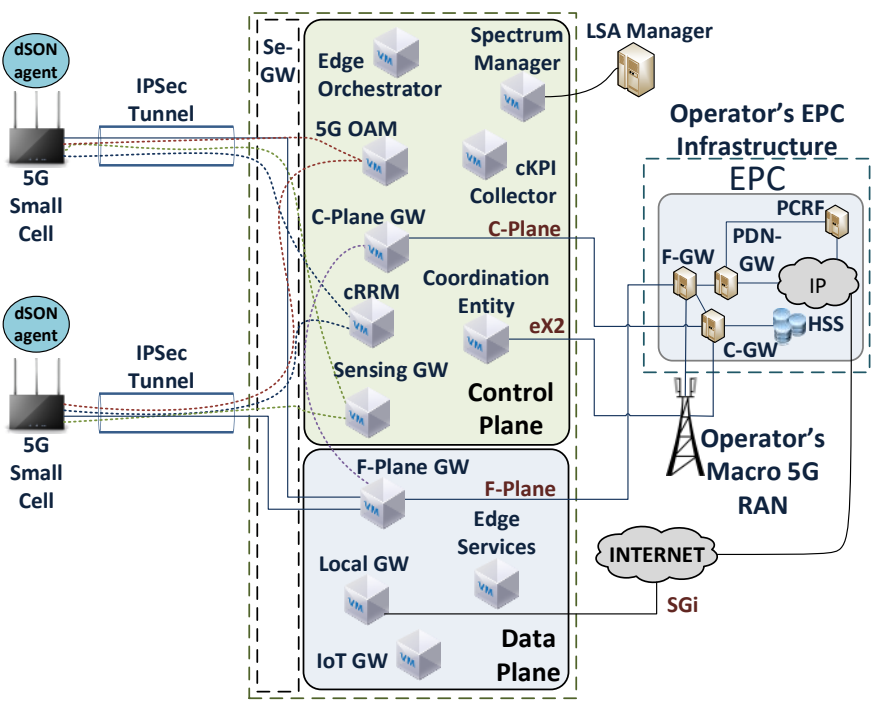

Fig. 3. SPEED-5G Edge Virtualized Architecture

The novel slice concept is based on deploying logical elements that can easily be defined, modified and started-up thanks to the principles of

i. Software-Defined Networks (SDN) [10] that allows to configure and instantiate the SW by decoupling control from data plane,

ii. Network Function Virtualization (NFV) [11] that supports virtual HW abstraction mechanisms, and

iii. Software-Defined Radio (SDR) [12] that supports the stack virtualization procedures. 
The final operator's network will be composed of a set of multiple slices covering different end user demands. Even if the slice concept is perfectly defined, the mobile network operators have not reached an agreement about its main goal, neither how to share physical resources using the slice concept.

Currently, two main approaches are being discussed: The first one defines that a slice covers a specific network user type as e.g. IoT or vehicular, consequently one slice may support several services. The second approach considers that a network is a union of slices, each one covering a specific service. Both solutions are a trade-off between network flexibility and complexity. SPEED-5G is aligned with the second approach where one service has to be covered by one slice. The solution increases the flexibility but, at same time, increases the SW and HW management complexity.

In any case, and based on the previously identified use cases, the proposed architecture reduces the control signalling, improves throughput, reduces latency for supporting real time services, enhances spectrum access without interfering with other systems, and finally provides flexibility, scalability and an easy interconnectivity with other networks.

Fig. 4 shows a specific SPEED-5G end-to-end network slice deployment for the previous eMBB use case. The slice is a concrete specification of the proposed architecture.

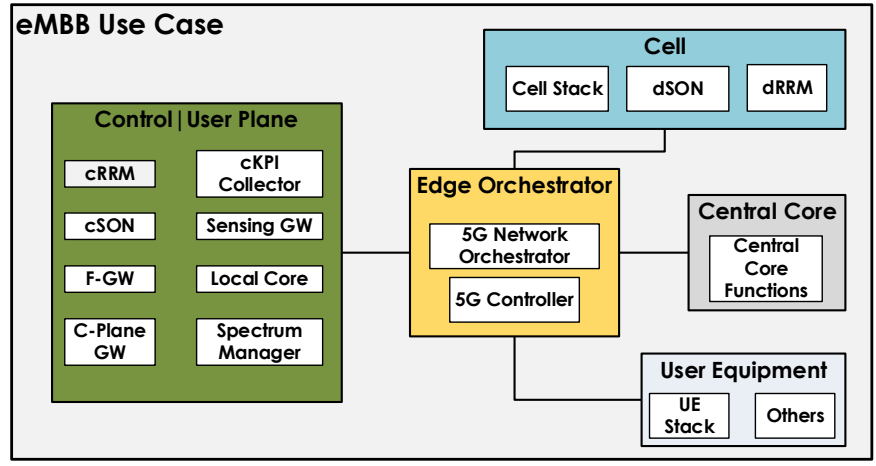

Fig. 4. SPEED-5G architecture for the eMBB use case

In this deployment, the slicing approach is one slice with a complete logical network that covers all end-to-end user requirements. Nevertheless, the proposed architecture is flexible enough to take another approach and provide a complete slice per eMBB service. The proposed slice is composed of the following logical associations: the Edge Orchestrator, the Central Core, the Control and User Data Plane, the Cell and the User Equipment.

\section{A. Edge Orchestrator}

It provides the automated arrangement, coordination, and management for SDN/NFV systems supporting flexibility, scalability, and service-oriented management coordinating between slices and between logical entities inside each slice.

- 5G Network Orchestrator: based on NFV, it refers to the software configuration, selection and allocation of network functions in a configurable protocol stack.
- 5G controller: based on SDN, it provides the APIs between all the network elements inside the slice and among the external slices.

\section{B. Central Core}

It contains the functions performed by the core entities as the Control Gateway $(\mathrm{GW})$, the Data Gateway, the Packet Data Network (PDN) GW or the Home Subscriber Server (HSS).

\section{Control and User Data Plane}

It contains all the logical entities dedicated to control and user data management:

- Centralized RRM (cRRM): it implements, in a centralized way, strategies and algorithms for mobility and link management, controlling parameters such as available operating bands, allowed resource allocation techniques, transmit power, data rates and modulation schemes for different services depending on its QoS.

- Control-Plane GW: it routes control data between cells, user equipment and core network.

- Centralized SON (cSON): it provides centralized SelfOrganizing Network (SON) solutions.

- Centralized KPI Collector: responsible to receive and process the KPI reports forwarded by the cells.

- Client Spectrum Manager: it is used for configuring the required spectrum measurements on the RRM.

- Local Core: executes the core functions that are moved from the central core into the slice, reducing latency and backhaul traffic volume.

- Forwarding GW (F-GW): it provides the anchor point for the user plane, routing the data services between the core network and the user equipment through the cells.

D. Cell

It contains logical entities, based on the SDR concept, that are usually associated to a BS (2G), a NB (3G) or an eNB (4G).

- Cell Stack: based on the SDR concept and, for the eMBB use case, contains the complete cell stack. Other use cases may require a centralized stack on the Control and User Plane logical association

- distributed RRM (dRRM): it executes functions that are not executed in the cRRM like user Congestion and Admission Control (CAC).

- distributed SON (dSON): it provides complementary cSON solutions ensuring high adaptability.

\section{E. User Equipment:}

It contains all logical functions related to the network terminals; it can be implemented as a smartphone or a sensor.

- UE Stack: the required UE communication protocol stacks (Wifi, cellular, etc.) that allow running the needed services, in this use case the eMBB-related ones. 
- Others: all other needed architectural blocks composing the parts of the UE outside of the protocol stacks (e.g., the location procedures).

The proposed SPEED-5G network architecture, in the eMBB use case, supports high data rates thanks to the used eDSA, coordinated by the Spectrum Manager and exploiting optimised RRM. Furthermore, end user service latency is improved by moving MBMS core functions into the Local Core of the relevant slice, avoiding additional backhaul delays. Additionally, the coordination between the cSON and the dSON algorithms ensure best network performance.

The flexibility of the SPEED-5G architecture allows to create slices for supporting eMBB services when required, and destroys them when not. The scalabilty of the slicing concept of the SPEED-5G architecture allows fast adaptation to user demands. Finally, as the architecture only deploys the required entities, it is lean and highly efficient.

The next section goes beyond this more functional level into the actual underlying resource management and required media access functions.

\section{RAdio RESOURCE MANAGEMENT}

A key component of the SPEED-5G architecture is the Radio Resource Manager (RRM). The RRM has the task of selecting the most appropriate radio access technology (RAT) to be used by each session, and tagging the session packets with the RAT reference. Fig. 5 shows that the RRM is positioned towards the top of the protocol stack and is centralised in the sense that it has an overview of many cells.

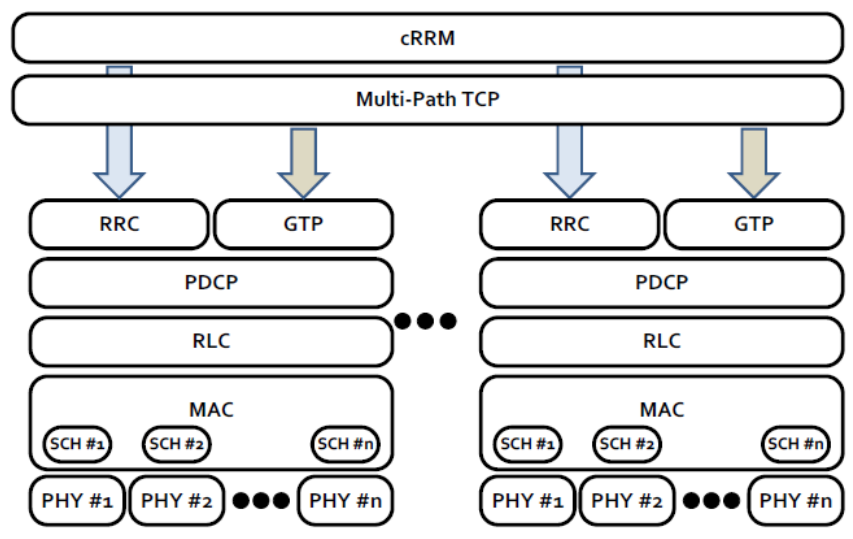

Fig. 5. The RRM has an overview of, and selects the RATs for, multiple cells

Multiple instances of cRRM will communicate with each other in order to cooperate successfully at the edges of their geographical coverage areas. The design includes a Multipath TCP layer, for scheduling TCP services over different radio bearers in parallel. These TCP paths are embedded in different GTP tunnels in order to re-direct these services to one or more RATs of a cell or of multiple cells, in order to support Cooperative Multi-Point (CoMP) and virtual multi-cell MIMO schemes. Every TCP packet is tagged according to the Multipath TCP protocol. UDP packets can be handled by wrapping in a Multipath TCP header, and dropping the ACKs.

Inputs to the RRM consist of a set of semi-static and a set of dynamically changing contextual information, as shown in
Fig. 6. The difference between semi-static and dynamic is the time-scales over which they are updated. The semi-static context is established by the Network Management system and updated infrequently, e.g. when the regulatory regime, system topology, or selection of KPIs change. The dynamic context can change many times per second, and is maintained through communications with the mobile network core for mobility and spectrum manager aspects, and through the base-station MAC/PHY layers for sensing and current performance aspects. The algorithms can interrogate the semi-static information whenever necessary, but the information flow is one-way.

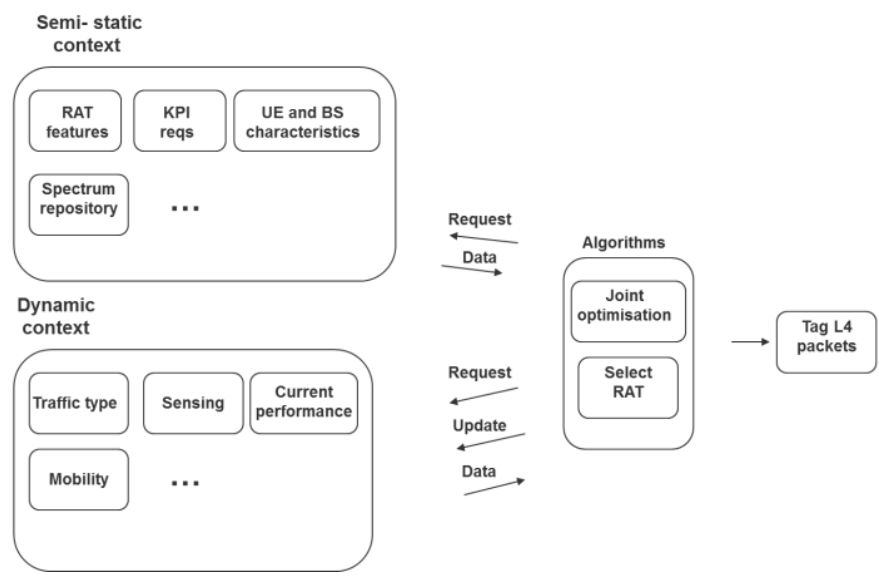

Fig. 6. The RRM algorithms take input from both semi-static and dynamic contexts

With the dynamic context, the algorithm actions will affect every one of the boxes, and a feedback mechanism is envisaged where the RAT selection will optimise the performance across all the traffic types, while putting the minimum load on the spectrum, transmit power and signalling overheads. Thus channel quality reports from UEs and small cells will be available as inputs to the RAT selection algorithm, as will be downlink and uplink bitrate requirements. The spectrum repository, which can be remote, contains rules for spectrum sharing, including whitespace. At all times a control channel to UEs will be kept open on the best currently available spectrum band.

\section{MULTI-RAT AND MAC TO IMPLEMENT E-DSA}

In literature, standardisation or industrial fora, Multi-RAT operation is getting quite a momentum as opportunistic resource allocation appears as a realistic short-term means to improve networks capacity. 3GPP e.g. progressively integrates aggregation of heterogeneous links as a complement to the traffic on licensed carrier: In Rel.10 and 12, LTE-U specification allows on the downlink to aggregate a primary carrier on licensed spectrum and a secondary carrier operated in the $5 \mathrm{GHz}$ band. Further developed at the LTE-U Forum, this carrier aggregation scheme provides a coexistence mechanism based on a time division with ON/OFF duty cycle of LTE-U cells [14]. In order to target geographic areas e.g. Europe, 3GPP has specified in Release 13 an LBT (ListenBefore-Talk) based version of LTE-U named LicensedAssisted Access (LAA) [15]. This is meant for ensuring a coexistence scheme in the $5 \mathrm{GHz}$ band compliant with the European regulatory framework [16]. Also a proper mechanism 
able to combine LTE and Wifi links in a heterogeneous carrier aggregation scheme called LTE-WLAN aggregation (LWA) is defined in Rel. 13.

Other standardisation groups like IEEE802.19 [17] address coexistence of multi-RAT unlicensed technologies, but extension into and coordination with licensed technologies have not been addressed so far. For example, the coexistence between IEEE802.x and LTE-U is still an open topic, even if research has defined general frameworks to classify scenarios [18] or looked at related cost functions [19].

Most 5G developments seem to follow an incremental approach building on evolutions of 3GPP standards, and this means that the opportunities to better exploit the available approaches in heterogeneous wireless technology (e.g. smart offload) or more dynamic access to spectrum, are not addressed in the most effective way, thus leading to a spectrum crunch limit forecast for licensed bands. The MAC protocol currently designed by the SPEED-5G project goes beyond that and will support multi-RAT resource allocation both in licensed and unlicensed/lightly-licensed bands

The SPEED-5G architecture was defined with the aim of meeting the headline $5 \mathrm{G}$ system KPIs. Looking in particular at the target of a 1000 times increase in mobile traffic volume, and therein the need to efficiently support a range of rather different service and traffic types, SPEED-5G targets a solution that relies on a combination of densification of small cells, efficient use of spectrum and exploiting multi-RAT resource management. Resource management is being applied in an effective way only to single technologies (e.g. LTE) but not to multi-RAT environments. At the core of the project is the definition of a new MAC layer that facilitates multi-RAT access, and allows prioritising and allocating traffic across heterogeneous access technologies.

SPEED-5G designs a system supporting e-DSA. This concept can be seen as a combination of two main drivers: a) heterogeneous resource management for inter-RAT cooperation and b) tight integration of advanced and flexible physical layer. Both should enable harvesting and aggregating fragmented spectrum with a smart access control designed to mitigate the interference in shared spectrum. From a MAC perspective, the first feature requires strong integration with advanced resource management and control of heterogeneous systems so that traffic offload is efficient while ensuring a satisfying QoE and protection of incumbent users in the case of shared spectrum. The second feature is designed to mitigate the interference in shared spectrum by aggregating fragmented spectrum with a smart access control. It exploits the relaxed constraints on orthogonality, synchronization and the low interference leakage of the Filter-Bank Multi Carrier (FBMC), which is one promising enabling technology chosen for an important part of the work at MAC layer. The configurability of such a physical layer makes it amenable to operate by aggregating various spectrum chunks while ensuring an efficient coexistence within adjacent radio systems in shared spectrum. SPEED-5G works on several techniques including resource management mechanisms, spectrum aggregation policies, etc. however this paper concentrates on the contributions for a MAC that can instantiate the Multi-RAT eDSA and RRM optimisation outcomes.
The SPEED-5G system has to exploit a flexible physical layer together with a MAC able to cope with heterogeneous QoS, inter-RAT operation, and interference management. Fig. 7 illustrates the SPEED-5G MAC where the main elements are the Configuration, the Scheduler, the Subframe, the KPI collector and the Sensed Data. The communication between MAC and contiguous layers is done through Service Access Points (SAP) denoted CMAC/MAC SAPs (for control and data paths) and PHY SAP. Similarly, communication between Scheduler and related functions of resource management and resource mapping functions are respectively done on SCHED SAP and CSCHED SAPs.

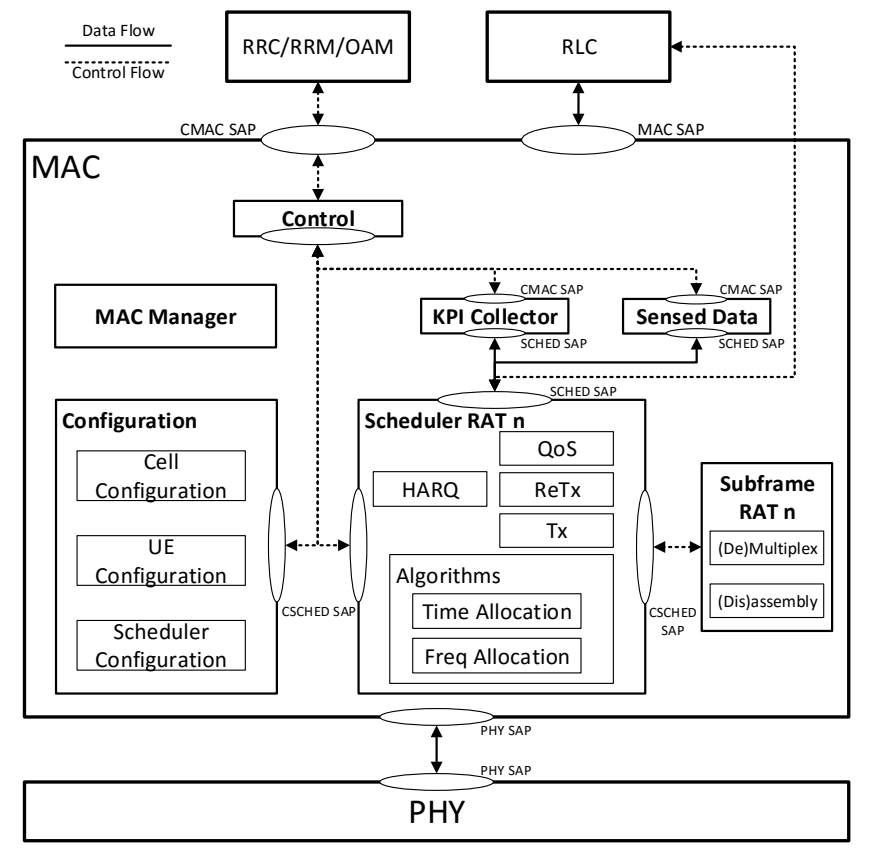

Fig. 7. MAC layer block diagram

The foreseen MAC layer includes several different MAC entities that provide features that are RAT-dependent, such as the scheduling process for resource allocation and the Hybrid Automatic Repeat Request (HARQ) management, when required. Therefore, the proposed MAC includes the capability to have a specific scheduler per RAT. Fig. 7 illustrates this, depicting only one of the supported schedulers (i.e. scheduler RAT \#n). Triggered by resource management and control decisions which select a given RAT, the "configuration" block activates the appropriate scheduler and provides configuration parameters e.g. for retransmission policy and Time/Frequency allocation rules. This configuration depends on aspects as QoS requirement, selected spectrum resource or SON decision upon interference context provided by the "KPI collector" block. The scheduler provides the "Subframe RAT n" block with the physical resource where logical channels have to be mapped and multiplexed and finally provided to the physical layer of RAT $n$.

As far as scheduling is concerned, operation in licensed or non-licensed resources has to be considered. For a licensed RAT like LTE, frequency allocation may be a well-known Round Robin or Proportional Fair scheduler which allocates, each Transmission Time Interval (TTI), the most suitable 
resource blocks of each schedulable UE. When operating in unlicensed or lightly licensed spectrum, the Time-Frequency allocation algorithms may be very different since the MAC layer can be contention-based (e.g. Wifi). In this case, the timefrequency allocation will be restricted to a channel or band identification, with no strict TTI management since frame duration sounds more relevant in this kind of spectrum bands. In the case of scheduled transmission in unlicensed or lightly licensed spectrum, the time-frequency allocation algorithms rely on the sensing context stored on the "Sensed Data" block to identify on a frame basis what will be the scheduled UEs (time allocation) on which frequency resource; the latter would be the whole channel (like in TDD scheme) or a subset if resource blocks can be allocated.

\section{CONCLUSIONS}

This paper describes and analyses requirements and KPIs of four key $5 \mathrm{G}$ use cases to be supported by a novel proposed $5 \mathrm{G}$ system architecture. The paper focuses on one of them, the eMBB use case, to show-case the advantages of the proposed $5 \mathrm{G}$ architecture. The foundations of such architecture are based on the Network Slicing paradigm using SDN/NFV technologies, which enables highly flexible, scalable, agile and backwards compatible novel 5G network architecture, allowing an enhanced QoS provision and a capacity expansion enabled through eDSA techniques for 5G. The basic concept of Radio Resource Management in a Multi-RAT environment has been sketched. Finally this paper provides a novel MAC layer able to support a seamless multi-RAT operation, where eDSA techniques are applied in order to increase the traffic volume while enhancing the QoE of the different users of the system.

The paper also presented the main results achieved so far but further work is still required and is already being performed on multiple areas in order to achieve the SPEED-5G goals. These include the specification and development of a MAC layer for the 5G Air Interface standard, enabling a Multi-RAT eDSA solution with native support for a new FBMC based air interface for $5 \mathrm{G}$ small cells; the development of new RRM mechanisms and higher layers intelligence for the optimal deployment of the eDSA function, including context-aware, service oriented RRM with dynamic allocation schemes, spectrum-aware hybrid SON algorithms, Control -/ Data Plane split management, enhanced cross-carrier scheduling, and in general for supporting the new SPEED-5G MAC Layer.

Future research activities in SPEED-5G will focus on new RRM algorithms required to coordinate and control new MAC layer functions depending on regulatory aspects related with the different available spectrum bands, UE capabilities or the specific network service; innovative MAC procedures and algorithms for ensuring optimal use of the available spectrum, deciding when orthogonal or non-orthogonal medium access is used depending on the use case, the considered network slice and frequency band. Those algorithms will also help deciding how the resources are split and managed across heterogeneous access technologies using unlicensed, licensed and license shared spectrum schemes, or configuring the frames for novel sensing procedures, thus improving the system performance using the same available resources.

\section{ACKNOWLEDGMENT}

The research leading to these results received funding from the European Union's Horizon 2020 research and innovation programme under grant agreement $n^{\circ} 671705$ (5G PPP project SPEED-5G). The paper reflects only the authors' views. The Commission is not responsible for any use that may be made of the information it contains.

\section{REFERENCES}

[1] "Mobile Data Traffic: Analysis of Demand vs Capacity", 5GIC internal report, 2014.

[2] SPEED-5G Website. Available online at http://speed-5g.eu

[3] P. Demestichas, A. Georgakopoulos, K. Tsagkaris, S. Kotrotsos, "Intelligent 5G networks: managing 5G wireless/mobile broadband", IEEE Vehicular Technology Magazine, Vol.10, No.3, pp.41-50, Sept. 2015.

[4] NGMN, "5G White Paper", available online at http://www.ngmn.org/home.html, accessed July 2015.

[5] FP7-METIS: "Scenarios, requirements and KPIs for 5G mobile and wireless system" Deliverable D1.1, FP7 METIS project. Document number ICT-317669-METIS/D1.1, May 2013. METIS project, official website: www.metis2020.com, accessed Jul. 2015.

[6] GSMA, "Powerful Digital Highways For Europe's Connected And Automated Driving", available online: http://www.gsma.com/newsroom/press-release/powerful-digitalhighways-for-europes-connected-and-automated-driving/, access. Feb.16.

[7] A. Fehske, G. Fettweis, J. Malmodin, and G. Biczok, "The Global Footprint of Mobile Communications: The Ecological and Economic Perspective," in IEEE Communications Magazine, vol. 49, no. 8, pp. 55 $-62,2011$.

[8] http://www.4gamericas.org/en/

[9] M. Iwamura, "NGMN View on 5G Architecture", IEEE Vehicular Technology Conference (VTC Spring), May 2015.

[10] H. Kim and N. Feamster, "Improving network management with software defined networking," IEEE Communications Magazine, vol. 51, no. 2, February 2013.

[11] ETSI ISG NFV (Operator Group), "Network Functions Virtualisation Network Operator Perspectives on Industry Progress", Updated White Paper, October 2014.

[12] J. Sachs and S. Baucke, "Virtual radio - A Framework for Configurable Radio Networks," International Conference on Wireless Internet (Wicon), October 2008.

[13] G. Wunder et al., "5GNOW: non-orthogonal, asynchronous waveforms for future mobile applications," in IEEE Communications Magazine, vol. 52, no. 2, pp. 97-105, February 2014.

[14] LTE-U Forum, "LTE-U Carrier Sensing Adaptive Transmission (CSAT) Procedure TS V1.0", October 2015.

[15] 3GPP "Study on Licensed Assisted Access to Unlicensed Spectrum", RAN Technical Specification Group, Release 13, June 2015

[16] ETSI EN 301 893, Broadband Radio Access Networks, 5 GHz high performance RLAN; Harmonized EN covering the essential requirements of artcile 3.2 of the RT\&TTE Directive, July 2014.

[17] A. Maeder, V. Mancuso, Y. Weizman, E. Biton, P. Rost, X. Perez-Costa, O. Gurewitz; FLAVIA: Towards a Generic MAC for $4 \mathrm{G}$ Mobile Cellular Networks, Future Network \& MobileSummit 2011 Conference Proceedings.

[18] J. Pérez-Romero, O. Sallent, R. Agustí, 2007, April. A generalized framework for multi-RAT scenarios characterisation. In Vehicular Technology Conference, 2007. VTC2007-Spring. IEEE 65th (pp. 980984). IEEE.

[19] EH Ong, JY Khan. On optimal network selection in a dynamic multiRAT environment. Communications Letters, IEEE. 2010 Mar;14(3):217-9 